\title{
Emerging service orientations and transformations (SOT)
}

\author{
Haluk Demirkan ${ }^{1}$ James C. Spohrer ${ }^{2}$
}

Published online: 19 May 2016

(C) Springer Science+Business Media New York 2016

Agility and innovation are essential for survival in today's business world. Mergers and acquisitions, new regulations, rapidly changing technology, increasing competition and heightened customer expectations mean companies must become more responsive to changing demands, i.e., become more agile. This move to agility through innovation has been referred to as the "innovation-services-based economy." If properly applied, "service orientation" and "service transformation" can support the agility organizations are seeking.

To achieve long term agility, companies must co-create their offerings with customers, and break siloed business processes into modular customer-centric independent services that can be reused on-the-fly in loosely-coupled dynamic business processes or "out-tasked" to external service providers that provides modularity and flexibility. On the other side, the sourcing of IT services needs to be aligned in a manner that takes cost advantage and flexibility of the commoditization of hardware (e.g., on-demand, utility computing, cloud computing, software oriented infrastructure with virtualized resources, infrastructure service providers), software (e.g. the software-as-service model, software oriented architecture, application service providers), and even business processes (e.g. out-tasking, ITIL, SCORE).

Basically, organizations are working two major initiatives: 1) service innovation, orientation and transformation to become more customer centric and how to break siloed

Haluk Demirkan

haluk@uw.edu

1 Milgard School of Business, University of Washington - Tacoma, 1900 Commerce Street, Box 358420, Tacoma, WA 98402-3100, USA

2 IBM Almaden Research Center, San Jose, CA 95120, USA business process to modular customer centric services; 2) how to source IT services with external services computing that will make them more flexible and agile to respond market needs much more efficiently and effectively.

These have been our prime motivation for this special issue, which was conceived as a vehicle for understanding some of today's issues and look at opportunities for in context of service orientation and transformation. We aimed to investigate this paradigm, review the impact of service and service innovations on federations of enterprises, organizational structures and individuals; investigate its tenets and evaluate relevant management and technical approaches to architecture, infrastructure, business processes, workflows and strategy.

Papers included in this Special Issue were selected and revised extensively from the work presented at the Third Australasian Conference on Service Research and Innovation held in Sydney, Australia on November 27-29, 2013. All six papers use theoretically grounded analysis to investigate a wide range of substantive issues and opportunities to increase outcomes of service orientation and transformation with service innovations and processes. We also emphasize the multidisciplinary nature of service innovation solutions by including studies that bring different theoretical background, in strategy, management, information systems and computer science. The authors' results in all of the articles have implications for services that go beyond the immediate application settings on which they report. These papers also showcase the application of an array of research methods, including surveys, experiments and design science. In the remainder of this Guest Editor's Introduction, we will briefly discuss each of the articles in the special issue to identify their main thrust of the authors' investigation and the relevant findings for theory and practice.

The first two papers provide a coherent characterization of the 'service system', 'service innovation" and 'service 
transformation' which constitutes the basic unit of analysis in the broader discourse on services and the service economy.

Service innovation and business model innovation are closely linked business strategy and performance. When we review some of the successful service innovations (e.g. Lego, IBM, Starbucks), we see that these customer-centric innovations are technology-enabled, human-centered and processoriented. In order for such sustainable innovations, organizations need an integrated cross disciplinary, holistic method to design and commercialize service innovation. In "iSIM: An Integrated Design Method for Commercializing Service Innovation," Chew (2016) develops a new integrated design method, known as iSIM (integrated Service Innovation Method), for simultaneous service innovation and business model design for sustained customer value co-creation with the firm. After providing a very comprehensive theoretical review from service science, strategic management, organization science and information systems literatures and by following the design science research method, this paper theoretically defines and integrates iSIM's seven constitutive design process-elements: service strategy, customer type / value proposition, service concept, service system, customer experience, service architecture and monetization into a coherent and endto-end aligned integrated design method. This framework has been evaluated by selected practitioners and its efficacy by two exemplar case studies using secondary data. Of course, two case studies are not valid points to effectively generalize this framework, but this is a great starting point for further research.

Many product organizations are in a process of transforming to become service-centric in order to provide better services to customers and also increase their revenue. Today, most organizations concentrate on the organizational changes rather than customer perspectives during these transformations. Marjanovic and Murthy's article titled "From Product-centric to Customer-centric Services in a Financial Institution - Exploring the Organizational Challenges of the Transition Process" (2016) describes an exploratory research case study conducted in a complex financial organization in the context of their customer services by using the Work Systems Theory. They found the key enabler of this process not in technology or strategy, but in much harder-to-grasp, let alone manage "service climate" consisting of employees" shared sense of service quality as stated in policies, practices and procedures, and the actual quality as observed in behaviors that are expected, supported and rewarded. This is one of the first studies that apply the Work Systems Theory in a real life example. This research makes several contributions to IS research. First, they advance the emergent IS thinking about the role of IS in service science research. By examining a transition process from productcentric to customer-centric services through the IS lens (supported by WST), they "bring services and service innovation to the foreground". They also contribute to the topic of "business/IT alignment" by demonstrating how this alignment should be continuous and rapidly evolving.

Second set of papers in this special issue investigates governance and compliance related issues of service orientation at organizations.

Research shows that the problems with SOA (ServiceOriented Architecture) governance in practice are among the major reasons of SOA failures. In order to address the existing challenges and accomplish a successful SOA adoption, organizations need exact definition of processes and relationships, control mechanisms, SOA metrics and enforcement of policies that are defined in a SOA governance framework. By adopting an interpretative research methodology, "SOA in Practice - A Study of Governance Aspects" by Joukhadar and Rabhi (2016) focuses on the role of the SOA aspects that are considered essential for a SOA governance framework. It empirically analyzes the position and role of each aspect and its importance to SOA governance according to SOA practitioners. The role of this framework is to focus on the architectural concepts, components and standards that are required to build effective SOA governance (Hojaji and Shirazi 2010). An extensive literature review that covers SOA, IT governance and SOA governance has been carried out part of this research. Based on a literature review, this study first proposes a list of SOA aspects to be considered when implementing SOA governance. The results provide a theoretical conceptualization of SOA aspects that can be used to assess SOA governance practices and provide guidance to improve them. While the literature is rich with SOA maturity models and related research, the importance of SOA vision has not been sufficiently acknowledged and not enough attention has been given to its role inside the organization and its relationship to other aspects specifically SOA maturity and SOA roadmap. The findings cited in this paper regarding the role of SOA aspects in building strong governance inside the organization would help organizations employing successful SOA governance from the beginning of the SOA implementation and not after the technology is applied.

In today's highly regulated corporate environment compliance has become an unavoidable activity for every enterprise. Enterprises, public and private alike, are adopting SOA based technologies to bring innovations into their business operations; and to offer their core competencies as Web Service (WS). Often physically independent, web services are collections of interrelated services orchestrated to provide a specific functionality; and web services are designed by combining disparate business processes from different enterprises. In such a dynamic setting, the ability to trust that one another's internal processes that form the core of successful invocation of web services are compliant with regulation becomes even more crucial. In the recent years several works addressed the 
issue of (regulatory) compliance in the context of of business process management, service computing and cloud computing domains (Becker et al. (2012); Fellmann and Zasada (2014) for recent surveys of existing approaches). "Normative Requirements for Regulatory Compliance: An Abstract Formal Framework" by Hashmi et al. (2016) offer a formal foundational framework to evaluate the ability of a compliance framework to representing the norms a system has to comply with. In this paper, they study the normative component of business process compliance and proposed an abstract formal framework comprising a classification of norms and formal semantics. In the classification, they examine various types of norms that can be imposed on different aspects of business processes in terms of temporal validity of a norms; and effects of violations on norms. They also provide formal semantics in terms of states determining the temporal validity, what constitutes a violation, effects of violations on other norms a process driven SOA system may be subject to, and the possible ways in which a business process can be executed. They used a complaint handling process and other case examples; and practically demonstrated how the compliance of business processes can be checked annotated with the compliance rules to validate the effectiveness of the proposed classification of normative requirements and compliance checking approach. This framework can be used in a number of ways: (1) it can be used to compare different systems, logics and frameworks for business process compliance. It can be used to study the (formal) properties of the problem of checking whether a business process is compliant.

The final set of two papers deal with emerging infrastructure services (such as infrastructure-as-a-service, cloud computing, data-as-a-service) in service-related research.

Enterprises considering transforming their IT services to services computing - cloud computing - have limited black box view of the offered infrastructure. While information about server pricing and service level agreements are publicly available most of the time, there is limited information about infrastructure performance. Cloud computing service providers use heterogeneous hardware resources, offer different server configurations, apply different pricing models and use different virtualization techniques to provision them. In the article "Smart CloudBench - A Framework for Evaluating Cloud Infrastructure Performance," Baruwal Chhetri et al. (2016) proposes a framework called to understand the runtime behavior of cloud infrastructure, helping companies to compare and contrast available offerings during the initial cloud selection phase and monitor performance for service quality assurance during the subsequent cloud consumption phase. This automated, on-demand, and customizable tool has been tested extensively and has the potential to take much of the guesswork out of cloud service acquisition decisions. The users of Smart CloudBench can design different types of experiments to test the performance of representative applications using load conditions that match the load levels of their own in-house applications.

Any of these service executions are very data intensive. More recently, event data analysis is becoming increasingly of interest to academic researchers looking for patterns in the data, contributing to the emergence and popularity of a new field called "data intensive science" (Rabhi et al. 2012). Chen and Rabhi (2016) "Enabling User-Driven Rule Management in Event Data Analysis," propose an approach (an architecture called EPRDR) to enable domain experts to manage event data analysis on historical data with minimal IT expert intervention by: Integrating a rule learning framework supporting incremental acquisition that enables domain users to define and add rules by themselves; Providing event pattern detection as a service (EPDaaS) in a way that allows domain users to conduct event processing without any concern about which event processing language/ engine to use. The proposed architecture is designed to enable management of the whole rule base, and to leverage the functionality of existing Event Processing Systems. With the involvement of RDR, domain experts can add a new rule only when they inspect an incorrect action, preventing the existing rule base from being corrupted. If domain experts intend conduct a different type of event data analysis, they can simply modify the rule set and alter the choice of the EPS invoked by the E DaaS component. This way, user-driven rule management is enabled with minimal technical support from IT experts, who are only involved in managing the EPL code in the database rather than the rule base. To validate the architecture, we have implemented a prototype mainly in Java. The EPDaaS component of the prototype calls two underlying EPSs. One is a simple EPS to perform initial validation, whereas the other one is a mature commercial EPS - EventSwarm. They have conducted a case study: event data cleansing in the context of financial data. A domain expert has successfully managed to evolve the rule base from 10 rules to 20 rules using event pattern types predefined by the IT experts. The proposed system has successfully addressed three evaluation criteria: feasibility and interoperability, complex event processing capability and user-driven rule set evolution capability.

Service orientation and transformation are enabling organizations to be agile and flexible, and to respond market changes much faster than used to. Organizations' IT is becoming much more flexible to join their value chain partners' IT networks to co-create value to the consumers (e.g. federated service oriented technology framework). Organizations are able to collect and process so much data they have never dreamed for with unstructured (e.g, Hadoop) and structured big data solutions (e.g. Big SQL).

SOT is becoming major enabler of smart machines. There is no doubt that computers are increasingly capable of doing things that humans could once do exclusively. Today smart machines are becoming like humans by recognizing voice, processing natural language, and interacting and learning with 
the physical world through their vision, smell, touch and other senses, mobility and motor control. In some cases, they do a much faster and better job than humans at recognizing patterns, performing rule based analysis from very large amounts of data and solving structured and unstructured problems. Being highly disruptive, service orientation and transformation will transform the way we work and live. Business processes and whole industries will be transformed, i.e., service oriented digital transformation.

The Digital Disruption Has Already Started

- Uber is the world's largest taxi company but owns no taxis (with cloud enabled mobile location based services)

- Airbnb is the largest accommodation provider but owns no real estate (with cloud enabled lodging services)

- Skype is one of the largest phone companies but owns no telco infrastructure (with cloud enabled communication services)

- Alibaba is the world's most valuable retailer but has no inventory (with cloud enabled retail services)

- Facebook is the most popular media owner but creates no content (with cloud enabled social network services)

- Netflix is the largest movie house but owns no cinemas (with cloud enabled entertainment services)

- Amazon is the largest retailer but produces no products or services (with cloud enabled retail services)

- MOOCs are the largest school but creates no course (with cloud enabled education services)

- Apple \& Google are the largest software vendors but do not write the apps (with cloud enabled mobile app retailers)

Changes will be required in employee workforce, corporate culture, partner ecosystems, law and regulations. As we conclude this project, we recognize that the research work that we have included in this special issue only scratches the surface of the issues that need to be studied. We expect enterprising future doctoral students and faculty to establish names for them by pursuing research agendas in services computing. We expect them to produce rich fundamental and applied work that leverages organizational and behavioral, economics and management science, and technical and design science research approaches toward the development of new managerial knowledge for service.

We know the importance of having to start "somewhere" to get the new ideas moving, and finding the appropriate collaborators to make some initial steps and advances in new knowledge possible. The guest editors would like to thank the Editors-in-Chief, R. Ramesh and H.R. Rao for the vision they shared with us. We also was fortunate to be able to assemble a highly committed group of authors whose papers were selected from among the many presented at ASRI 2013 (http:// www.servicesciencesociety.org.au/third-australasiansymposium-on-service-research-and-innovation-2/).
We would especially like to acknowledge the editorial board members (Joseph G. Davis, University of Sydney; Aditya Ghose, University of Wollongong; Hamid R. Motahari-Nezhad, IBM Almaden Research Center, USA; Fethi Rabhi, University of New South Wales), anonymous reviewers, who so generously offered their time, effort and helpful insights for us to make the hard choice and for helping me with development and constructive reviewing that led to the final products that you see in the present special issue. Finally, I thank the authors, including those whose works we accepted, and those whose efforts did not permit their research to go the final distance to publication. They all were diligent and careful, and gave us private lessons along the way about what vibrant and creative research on service science already looks like in the present. We look forward to the "next generation" of submissions to $I S F$, and hope that future authors will build on the foundations that we have established here.

\section{References}

Baruwal Chhetri, M., Chichin, S., Vo, Q. B., \& Kowalczyk, R. (2016). Smart CloudBench-A framework for evaluating cloud infrastructure performance. Information Systems Frontiers, 18(3). doi:10. 1007/s10796-015-9557-2.

Becker, J., Delfmann, P., Eggert, M., \& Schwittay, S. (2012). Generalizability and Applicability of Model-Based Business Process Compliance-Checking Approaches - A Stateof- the-Art Analysis and Research Roadmap. BuR - Business Research Journal, 5(2), 221-247.

Chen, W. \& Rabhi, F. (2016). Enabling user-driven rule management in event data analysis. Information Systems Frontiers, 18(3). doi:10. 1007/s10796-016-9633-2.

Chew, E. K. (2016). iSIM: An integrated design method for commercializing service innovation. Information Systems Frontiers, 18(3). doi: 10.1007/s10796-015-9605-y.

Fellmann M, Zasada A (2014). State-of-the-Art of Business Process Compliance Approaches. In: 22st European Conference on Information Systems, ECIS 2014, Tel Aviv, Israel, June 9-11, 2014, URL http://aisel.aisnet.org/ecis2014/ proceedings/track06/8

Hashmi, M., Governatori, G., \& Wynn, M. T. (2016). Normative requirements for regulatory compliance: An abstract formal framework. Information Systems Frontiers, 18(3). doi:10.1007/s10796-0159558-1.

Hojaji, F., \& Shirazi, M. R. A. (2010). 16-18 April 2010. Developing a more comprehensive and expressive SOA governance framework. Paper presented at the Information Management and Engineering (ICIME), 2010 The 2nd IEEE International Conference on.

Joukhadar, G., \& Rabhi, F. (2016). SOA in practice - a study of governance aspects. Information Systems Frontiers, 18(3). doi:10.1007/ s10796-015-9607-9.

Marjanovic, O., \& Murthy, V. (2016). From product-centric to customercentric services in a financial institution - exploring the organizational challenges of the transition process. Information Systems Frontiers, 18(3). doi:10.1007/s10796-015-9606-x.

Rabhi, F. A., Yao, L., \& Guabtni, A. (2012). ADAGE: A Framework for Supporting User- Driven Ad-hoc Data Analysis Processes. Computing, 94(6), 489-519. doi:10.1007/s00607-012-0193-0. 
Haluk Demirkan is a Professor of Service Innovation \& Business Analytics at the Milgard School of Business, University of Washington Tacoma. His doctorate is in Information Systems and Operations Management from University of Florida, and his research in analytics, digital \& service innovation and service-oriented technology \& management have included recent industry-sponsored research projects with IBM, American Express, Intel and Teradata. He has almost 20 years of professional work experience on maximizing the return on the companies' resources by effectively implementing strategic data and analytic solutions for 40+ Fortune 500 companies. He has more than 150 publications. He is a board of director for the International Society of Service Innovation Professionals, and an advisory board member for the INFORMS Service Science Section.
Jim Spohrer is IBM Innovation Champion and Director of IBM University Programs (IBM UP) and Cognitive Institute. Jim works to align IBM and universities globally for innovation amplification. Previously, Jim helped to found IBM's first Service Research group, the global Service Science community, and was founding CTO of IBM's Venture Capital Relations Group in Silicon Valley. During the 1990's while at Apple Computer, he was awarded Apple's Distinguished Engineer Scientist and Technology title for his work on next generation learning platforms. Jim has a PhD in Computer Science/ Artificial Intelligence from Yale, and BS in Physics from MIT. His current research priorities include applying service science to study nested, networked holistic service systems, such as cities and universities. He has more than 90 publications and been awarded nine patents. 